\title{
Genetic variants in the TGF $\beta$-signaling pathway influence expression of miRNAs in colon and rectal normal mucosa and tumor tissue
}

\author{
Martha L. Slattery ${ }^{1}$, Andromahi Trivellas ${ }^{2}$, Andrew J. Pellatt ${ }^{2}$, Lila E. Mullany ${ }^{1}$, John \\ R. Stevens ${ }^{3}$, Roger K. Wolff ${ }^{1}$, Jennifer S. Herrick ${ }^{1}$ \\ ${ }^{1}$ Department of Medicine, University of Utah, Salt Lake City, Utah, USA \\ ${ }^{2}$ Tulane Medical School, New Orleans, Louisiana, USA \\ ${ }^{3}$ Department of Mathematics and Statistics, Utah State University, Logan, Utah, USA \\ Correspondence to: Martha L. Slattery, email: marty.slattery@hsc.utah.edu \\ Keywords: colorectal cancer, miRNA, TGF- $\beta$, elF4E, SMAD \\ Received: October 13, $2016 \quad$ Accepted: December 16, $2016 \quad$ Published: January 05, 2017
}

\section{ABSTRACT}

The TGF- $\beta$ signaling pathway is involved in regulation of cell growth, angiogenesis, and metastasis. We test the hypothesis that genetic variation in the TGF- $\beta$ signaling pathway alters miRNA expression.

We use data from 1188 colorectal cancer cases to evaluate associations between 80 SNPs in 21 genes.

Seven variants eIF4E rs12498533, NFKB1 rs230510, TGFB1 rs4803455, TGFBR1 rs1571590 and rs6478974, SMAD3 rs3743343, and RUNX1 rs8134179 were associated with expression level of miRNAs in normal colorectal mucosa. RUNX2 rs12333172 and BMPR1B rs13134042 were associated with miRNAs in normal colon mucosa; eIF4EBP3 rs250425, SMAD3 rs12904944, SMAD7 rs3736242, and PTEN rs532678 were associated with miRNA expression in normal rectal mucosa. Evaluation of the differential expression between carcinoma and normal mucosa showed that SMAD3 rs12708491 and rs2414937, NFKB1 rs230510 and rs3821958, and RUNX3 rs6672420 were associated with several miRNAs for colorectal carcinoma. Evaluation of site-specific differential miRNA expression showed that BMPR1B rs2120834, BMPR2 rs2228545, and eIF4EBP3 rs250425 were associated with differential miRNA expression in colon tissue and SMAD3 rs12901071, rs1498506, and rs2414937, BMPR2 rs2228545, and $R U N X 2$ rs2819854, altered differential miRNA expression in rectal tissue.

These data support the importance of the TGF- $\beta$ signaling pathway to the carcinogenic process, possibly through their influence on miRNA expression levels.

\section{INTRODUCTION}

The TGF- $\beta$ signaling pathway is an essential regulator of cellular proliferation, differentiation, apoptosis, extracellular matrix remodeling in the cell, and is involved in angiogenesis and inflammation $[1,2]$. Components of this pathway have been associated with colorectal cancer risk and survival [3-8]. There are several components of the TGF- $\beta$ signaling pathway. Smads are key intracellular mediators of the transcriptional responses to TGF- $\beta$ [9]. Bone morphogenetic proteins (BMP), trigger a Smad-signaling cascade that is linked to reduced cell proliferation and cellular growth kinetics of glioblastomas $[10,11]$. The Runtrelated transcription factors (RUNX), RUNX1, RUNX2, and $R U N X 3$ [12] are involved in signaling cascades mediated by TGF- $\beta$ and BMP [13-16]. All three of the RUNX genes have been shown to bind Smads [17-19]. Studies in RUNX3 knockout mice have shown defects in apoptotic response to TGF- $\beta$; RUNX2 transgenic mice were hypersensitive to TGF- $\beta$ in one study [14]. Mitogen-activated protein kinase 1 (MAPK1) activates RUNX2 [20] and is involved in the TGF- $\beta$-signaling pathway through its role in Smad signaling [21, 22]. Eukaryotic translation initiation factor $4 \mathrm{E}$ (eIF4E) is a translational regulator; expression of eIF4E in human colon cancer cells promotes the TGF $\beta$ 


\begin{tabular}{|c|c|c|c|c|}
\hline & \multicolumn{2}{|c|}{ Colon } & \multicolumn{2}{|c|}{ Rectal } \\
\hline & $\mathbf{N}$ & $\%$ & $\mathbf{N}$ & $\%$ \\
\hline \multicolumn{5}{|l|}{ Center } \\
\hline Kaiser & 509 & 73.9 & 302 & 60.5 \\
\hline Utah & 180 & 26.1 & 197 & 39.5 \\
\hline \multicolumn{5}{|l|}{ Sex } \\
\hline Male & 383 & 55.6 & 296 & 59.3 \\
\hline Female & 306 & 44.4 & 203 & 40.7 \\
\hline \multicolumn{5}{|l|}{ Race } \\
\hline White, non-Hispanic & 594 & 86.2 & 400 & 80.2 \\
\hline Hispanic & 47 & 6.8 & 46 & 9.2 \\
\hline Black, non-Hispanic & 46 & 6.7 & 18 & 3.6 \\
\hline Other/Unknown & 2 & 0.3 & 35 & 7.0 \\
\hline \multicolumn{5}{|l|}{ Vital Status ${ }^{1}$} \\
\hline Dead & 279 & 40.6 & 184 & 36.9 \\
\hline Alive & 409 & 59.4 & 315 & 63.1 \\
\hline \multicolumn{5}{|l|}{ AJCC Stage $^{2}$} \\
\hline 1 & 169 & 24.8 & 227 & 46.0 \\
\hline 2 & 221 & 32.4 & 94 & 19.0 \\
\hline 3 & 226 & 33.1 & 142 & 28.7 \\
\hline \multirow[t]{2}{*}{4} & 66 & 9.7 & 31 & 6.3 \\
\hline & Mean & SD & Mean & SD \\
\hline Age & 65.3 & 9.1 & 61.8 & 11.1 \\
\hline Survival Months & 69.3 & 32.7 & 67.6 & 27.2 \\
\hline
\end{tabular}

${ }^{1}$ Vital Status missing for one individual.

${ }^{2}$ AJCC stage unknown for 12 people.

stimulation of adhesion molecules [23]. Other genes, such as nuclear factor kappa B1 (NFkB1), mammalian target of rapamycin (mTOR), and phosphatase tensin homolog deleted on chromosome 10 (PTEN), are major regulators of inflammation, are associated with colorectal cancer, and influence the TGF-B signaling pathway [24-26].

The TGF- $\beta$ signaling pathway has been shown to suppress growth and tumorigenicity in a normal cellular environment; but, in neoplastic cells, its role converts to the promotion of invasion and metastasis [27]. It has recently been hypothesized that miRNAs may be intermediaries in a TGF $\beta$-miRNA pathway that both mediates cell growth and promotes invasive behavior [28]. Some studies have linked miRNAs and the TGF- $\beta$ signaling pathway. For instance miR-181a has its expression upregulated by TGF $\beta$, which in turn promotes breast cancer metastasis $[28,29]$. Other studies have shown that TGF $\beta$ activates AKT kinase through mechanisms involving miRNAs, and that TGF $\beta$-signaling may be involved in the control of miRNA biogenesis [30, 31].

In this paper, we test the hypothesis that genetic variation in the TGF- $\beta$ signaling pathway is associated with miRNA expression level. We use data from a large study of colorectal cancer cases. We focus on genetic variants in 21 genes in the TGF- $\beta$ signaling pathway that we have previously shown to be associated with either colon or rectal cancer risk or survival after diagnosis. Our ability to show that these genetic variants alter miRNA expression adds support to the importance of these SNPs in cancer risk.

\section{RESULTS}

The majority of cases for both colon and rectal cancer studies were male (Table 1). The mean age at diagnosis was slightly older for those individuals enrolled in the colon cancer study compared to those enrolled in the 
rectal cancer study. Over $80 \%$ of the study population was non-Hispanic white. The majority of cases were diagnosed at either an AJCC stage 1 or stage 2.

MiRNA expression level differed across genotypes of seven SNPs, eIF4E rs12498533, NFKB1 rs230510, TGFB1 rs4803455, TGFBR1 rs1571590 and rs6478974, SMAD3 rs3743343, and RUNX1 rs8134179 in normal colorectal mucosa (Table 2). All but four miRNAs, miR4324, miR-4655-3p, miR-3663-3p, and miR-1539 that were significantly different by both TGFBR1 rs 1571590 and TGFBR1 rs6478974 when considering an FDR of $<0.09$ (Supplementary Table 1 for all associations). However, the majority of miRNAs associated with rs6478974 were downregulated for the variant allele, while for rs 1571590 the miRNAs were equally up and downregulated for the variant allele. For EIF4E rs12498533, NFKB1 rs230510, SMAD3 rs3743343, $T G F B 1$ rs4803455, all miRNAs were downregulated in the presence of the variant allele. However, for RUNX1 rs8134179 all of the miRNAs were upregulated in the variant allele.

Four SNPs, RUNX2 rs12333172, eIF4E rs12498533, $B M P R 1 B$ rs13134042, and RUNX1 rs8134179 were associated predominately with increased miRNA expression in normal colonic mucosa for the variant allele. Six SNPs were associated with miRNA expression in normal rectal mucosa: SMAD3 rs12904944, TGFBR1 rs1571590, eIF4EBP3 rs250425, SMAD7 rs3736242, and PTEN rs532678, and TGFBR1 rs6478974 (Table 3). SNPs in TGFBR1 were associated with expression of miRNAs for colorectal normal mucosa overall as well as for rectal normal mucosa specifically. eIF4E rs 12498533 was associated with downregulation of miR-221-3p in overall colorectal mucosa and also was associated with upregulation of miR-3180-3p in normal colonic mucosa. $R U N X 1$ rs8134179 was associated with miR-658 in a similar manner for colorectal (Supplemental Table 2 For all miRNAs associated with TGFBeta signaling pathway SNPs in normal colonic mucosa and Supplemental Table 3 for associations in normal rectal mucosa) normal mucosa overall and colon-specific normal mucosa.

Differences in expression between carcinoma and normal mucosa, or differential miRNA expression by genotype, was associated with six SNPs, SMAD3 rs12708492, BMPR2 rs228545, and $N F \kappa B 1$ rs230510, SMAD3 rs2414937, NFKB1 rs3821958, and RUNX3 rs6672420 (Table 4) for colorectal cancer overall. SMAD3 rs12708492 was associated with differential miRNA expression of six miRNAs miR-23a-5p, miR-30c-2$3 \mathrm{p}$, miR-4286, miR-4753-5p, miR-4753-3p and miR4679-5p by genotype. All miRNAs were downregulated with the variant allele except for miR-4286, where the expression was upregulated in the presence of the variant allele. SMAD3 rs2414937 was associated with only one miRNA, miR-590-5p, and it was upregulated among those with the variant allele. $N F \kappa B 1$ rs230510 was associated with expression of several miRNAs, all of which were downregulated for the variant allele, however the miRNAs associated with $N F \kappa B 1$ rs3821958 were upregulated. Interestingly miR-4684-3p was upregulated in the variant allele of $N F \kappa B 1$ rs230510 while downregulated in the presences of the variant allele of $N F \kappa B 1 \mathrm{rs} 3821958$.

Four SNPs, BMPR1B rs12508087, BMPR1B rs2120834, BMPR2 rs2228545, and eIF4EBP3 rs250425 were associated with differences in differential miRNA expression for colon tissue (Table 5). The majority of miRNAs associated with BMPR1B rs12508087 and rs2120834, and for BMPR2 rs2228545 showed upregulated differential expression. Supplemental Table 4 shows all miRNAs whose differential expression was associated with TGFBeta signaling pathway SNPs in colon tissue for the variant allele of eIF4EBP 3 rs 250425 were downregulated.

SMAD3 rs12901071, rs1498506, and rs2414937 were associated with differential miRNA expression in rectal tissue; however, only one miRNA was dysregulated for both rs12901071 and rs2414937. MiR-17-5p was upregulated by SMAD3 rs12901071 and downregulated by $S M A D 3$ rs2414937 (Table 6). Additionally, BMPR2 rs2228545 and RUNX2 rs2819854 genotypes were associated with differential miRNA expression between rectal carcinoma and normal rectal mucosa. Both miR324-5p and miR-484 were upregulated for the variant allele of BMPR2 rs2228545 while all but two miRNAs were (Supplemental Table 5 shows associations with TGFBeta signaling pathway SNPs for all differentially expressed miRNAs in rectal tissue) downregulated in the variant allele of $R U N X 2 \mathrm{rs} 2819854$.

\section{DISCUSSION}

The TGF- $\beta$-signaling pathway is one of the most important pathways involved in colorectal cancer development [32]. The TGF- $\beta$ signaling pathway is involved in maintaining normal cell growth in a nontumor cellular environment; alternatively, it can promote invasion and metastasis in neoplastic cells [27]. It has been proposed that miRNAs can act as intermediaries in a TGFB-miRNA pathway that both mediates cell growth and promotes invasive behavior [28]. Our data lend support to this dual role of the TGF- $\beta$ signaling pathway. Distinct SNPs and associated miRNA expression were observed for normal colorectal mucosa and for differential expression of miRNAs between carcinoma and normal mucosa. MiRNA expression in normal mucosa reflects a non-tumor environment and the role of the TGF- $\beta$ signaling pathway in maintaining normal cell growth. However, when examining differential expression between carcinoma and normal mucosa, associations suggest that the miRNA expression is more related to tumor promotion and possible metastatic potential. 
Table 2: Associations between TGFB-pathway SNPs and miRNA differential expression in colorectal tissue

\begin{tabular}{|c|c|c|c|c|c|}
\hline miRNA & Mean & Mean & P-values & FDR adjusted $P$ & Direction \\
\hline $\boldsymbol{E I F 4 E}(\mathrm{rs} 12498533)$ & AA $(\mathrm{N}=342)$ & $\mathrm{AC} / \mathrm{CC}(\mathrm{N}=798)$ & & & \\
\hline hsa-miR-221-3p & 3.21 & 2.25 & $<.0001$ & 0.0820 & Downregulated \\
\hline TGFBR1 (rs1571590) & $\mathrm{AA}(\mathrm{N}=738)$ & $\mathrm{AG} / \mathrm{GG}(\mathrm{N}=372)$ & & & \\
\hline hsa-miR-100-5p & 7.83 & 6.06 & $<.0001$ & 0.0373 & Downregulated \\
\hline hsa-miR-130a-3p & 3.20 & 2.26 & 0.0004 & 0.0373 & Downregulated \\
\hline hsa-miR-143-3p & 7.23 & 5.89 & 0.0004 & 0.0373 & Downregulated \\
\hline hsa-miR-19b-3p & 8.53 & 6.67 & 0.0003 & 0.0373 & Downregulated \\
\hline hsa-miR-29c-3p & 15.56 & 13.19 & 0.0005 & 0.0373 & Downregulated \\
\hline hsa-miR-3666 & 19.31 & 20.74 & $<.0001$ & 0.0373 & Upregulated \\
\hline hsa-miR-4324 & 1.30 & 0.98 & 0.0002 & 0.0373 & Downregulated \\
\hline hsa-miR-4655-3p & 22.54 & 24.35 & 0.0005 & 0.0373 & Upregulated \\
\hline hsa-miR-4659a-3p & 27.57 & 29.81 & 0.0003 & 0.0373 & Upregulated \\
\hline hsa-miR-5003-3p & 18.59 & 20.12 & 0.0005 & 0.0373 & Upregulated \\
\hline hsa-miR-6500-5p & 18.18 & 19.53 & 0.0005 & 0.0373 & Upregulated \\
\hline hsa-miR-662 & 41.11 & 43.73 & 0.0006 & 0.0410 & Upregulated \\
\hline hsa-miR-140-3p & 7.16 & 6.09 & 0.0008 & 0.0492 & Downregulated \\
\hline hsa-miR-26a-5p & 86.12 & 75.56 & 0.0009 & 0.0492 & Downregulated \\
\hline hsa-miR-4294 & 21.48 & 22.41 & 0.0009 & 0.0492 & Upregulated \\
\hline NFKB1 (rs230510) & AA $(\mathrm{N}=387)$ & AT/TT $(\mathrm{N}=723)$ & & & \\
\hline hsa-miR-4446-3p & 16.16 & 13.90 & $<.0001$ & 0.0820 & Downregulated \\
\hline SMAD3 (rs3743343) & $\mathrm{TT} / \mathrm{TC}(\mathrm{N}=1035)$ & $\mathrm{CC}(\mathrm{N}=75)$ & & & \\
\hline hsa-miR-1285-3p & 19.98 & 17.98 & $<.0001$ & 0.0410 & Downregulated \\
\hline hsa-miR-3935 & 16.98 & 14.78 & $<.0001$ & 0.0410 & Downregulated \\
\hline TGFB1 (rs4803455) & $\mathrm{CC}(\mathrm{N}=275)$ & $\mathrm{CA} / \mathrm{AA}(\mathrm{N}=881)$ & & & \\
\hline hsa-miR-146b-5p & 1.48 & 0.92 & 0.0002 & 0.0820 & Downregulated \\
\hline hsa-miR-3609 & 1.68 & 1.20 & $<.0001$ & 0.0820 & Downregulated \\
\hline TGFBR1 (rs6478974) & TT $(\mathrm{N}=359)$ & $\mathrm{TA} / \mathrm{AA}(\mathrm{N}=750)$ & & & \\
\hline hsa-miR-1226-5p & 54.62 & 51.50 & 0.0011 & 0.0355 & Downregulated \\
\hline hsa-miR-151a-5p & 7.57 & 9.03 & 0.0011 & 0.0355 & Upregulated \\
\hline hsa-miR-1587 & 1405.23 & 1292.11 & 0.0013 & 0.0355 & Downregulated \\
\hline hsa-miR-199a-3p & 16.51 & 19.29 & 0.0005 & 0.0355 & Upregulated \\
\hline hsa-miR-200a-3p & 18.50 & 20.89 & 0.0006 & 0.0355 & Upregulated \\
\hline hsa-miR-20a-5p & 13.21 & 15.67 & 0.0016 & 0.0355 & Upregulated \\
\hline hsa-miR-214-3p & 4.76 & 5.65 & 0.0005 & 0.0355 & Upregulated \\
\hline hsa-miR-222-3p & 8.28 & 8.99 & 0.0016 & 0.0355 & Upregulated \\
\hline hsa-miR-22-3p & 12.78 & 14.57 & 0.0007 & 0.0355 & Upregulated \\
\hline
\end{tabular}




\begin{tabular}{|c|c|c|c|c|c|}
\hline miRNA & Mean & Mean & P-values & FDR adjusted $P$ & Direction \\
\hline hsa-miR-25-3p & 8.70 & 10.46 & 0.0016 & 0.0355 & Upregulated \\
\hline hsa-miR-26b-5p & 15.33 & 17.83 & 0.0004 & 0.0355 & Upregulated \\
\hline hsa-miR-30c-5p & 7.73 & 9.17 & 0.0012 & 0.0355 & Upregulated \\
\hline hsa-miR-3156-5p & 95.91 & 90.85 & 0.0009 & 0.0355 & Downregulated \\
\hline hsa-miR-3185 & 17.99 & 16.77 & 0.0005 & 0.0355 & Downregulated \\
\hline hsa-miR-33b-3p & 19.25 & 15.53 & 0.0006 & 0.0355 & Downregulated \\
\hline hsa-miR-3620-5p & 95.85 & 89.60 & 0.0015 & 0.0355 & Downregulated \\
\hline hsa-miR-3621 & 38.13 & 35.90 & 0.0015 & 0.0355 & Downregulated \\
\hline hsa-miR-370 & 41.68 & 39.44 & 0.0002 & 0.0355 & Downregulated \\
\hline hsa-miR-3917 & 99.12 & 93.52 & 0.0005 & 0.0355 & Downregulated \\
\hline hsa-miR-3934-3p & 29.59 & 28.27 & 0.0014 & 0.0355 & Downregulated \\
\hline hsa-miR-3937 & 70.06 & 65.67 & 0.0013 & 0.0355 & Downregulated \\
\hline hsa-miR-4253 & 46.35 & 43.92 & 0.0012 & 0.0355 & Downregulated \\
\hline hsa-miR-4294 & 22.41 & 21.50 & 0.0011 & 0.0355 & Downregulated \\
\hline hsa-miR-4535 & 70.15 & 66.72 & 0.0007 & 0.0355 & Downregulated \\
\hline hsa-miR-4655-5p & 34.25 & 32.23 & 0.0013 & 0.0355 & Downregulated \\
\hline hsa-miR-4695-5p & 385.66 & 365.84 & 0.0009 & 0.0355 & Downregulated \\
\hline hsa-miR-4726-5p & 22.13 & 21.13 & 0.0015 & 0.0355 & Downregulated \\
\hline hsa-miR-4734 & 210.70 & 198.06 & 0.0013 & 0.0355 & Downregulated \\
\hline hsa-miR-5196-5p & 63.33 & 58.28 & 0.0012 & 0.0355 & Downregulated \\
\hline hsa-miR-550a-3-5p & 28.29 & 26.77 & 0.0009 & 0.0355 & Downregulated \\
\hline hsa-miR-564 & 67.17 & 64.76 & 0.0014 & 0.0355 & Downregulated \\
\hline hsa-miR-601 & 46.91 & 44.06 & 0.0010 & 0.0355 & Downregulated \\
\hline hsa-miR-6084 & 18.25 & 16.81 & 0.0012 & 0.0355 & Downregulated \\
\hline hsa-miR-610 & 25.93 & 24.45 & 0.0013 & 0.0355 & Downregulated \\
\hline hsa-miR-6511b-5p & 65.88 & 60.79 & 0.0005 & 0.0355 & Downregulated \\
\hline hsa-miR-760 & 34.80 & 32.70 & 0.0014 & 0.0355 & Downregulated \\
\hline hsa-miR-93-5p & 10.92 & 12.98 & $<.0001$ & 0.0355 & Upregulated \\
\hline hsa-miR-15b-5p & 20.02 & 23.05 & 0.0019 & 0.0359 & Upregulated \\
\hline hsa-miR-3187-3p & 9.99 & 9.16 & 0.0021 & 0.0359 & Downregulated \\
\hline hsa-miR-4646-5p & 112.80 & 106.87 & 0.0021 & 0.0359 & Downregulated \\
\hline hsa-miR-4738-3p & 50.24 & 48.07 & 0.0020 & 0.0359 & Downregulated \\
\hline hsa-miR-4740-5p & 33.39 & 31.96 & 0.0017 & 0.0359 & Downregulated \\
\hline hsa-miR-5189 & 33.40 & 31.42 & 0.0021 & 0.0359 & Downregulated \\
\hline hsa-miR-550b-2-5p & 38.12 & 35.90 & 0.0020 & 0.0359 & Downregulated \\
\hline hsa-miR-6124 & 522.71 & 487.27 & 0.0018 & 0.0359 & Downregulated \\
\hline hsa-miR-6165 & 323.94 & 306.32 & 0.0021 & 0.0359 & Downregulated \\
\hline hsa-miR-6500-5p & 19.30 & 18.32 & 0.0019 & 0.0359 & $\begin{array}{l}\text { Downregulated } \\
\text { (Continued) }\end{array}$ \\
\hline
\end{tabular}




\begin{tabular}{|c|c|c|c|c|c|}
\hline miRNA & Mean & Mean & P-values & FDR adjusted $P$ & Direction \\
\hline hsa-miR-671-5p & 291.07 & 271.09 & 0.0018 & 0.0359 & Downregulated \\
\hline hsa-miR-6722-3p & 103.52 & 97.67 & 0.0022 & 0.0368 & Downregulated \\
\hline hsa-miR-196b-5p & 3.53 & 4.76 & 0.0023 & 0.0370 & Upregulated \\
\hline hsa-miR-4481 & 84.65 & 78.48 & 0.0023 & 0.0370 & Downregulated \\
\hline hsa-miR-4758-5p & 131.05 & 120.75 & 0.0024 & 0.0378 & Downregulated \\
\hline hsa-miR-1247-3p & 26.28 & 25.11 & 0.0028 & 0.0396 & Downregulated \\
\hline hsa-miR-199a-5p & 6.22 & 7.34 & 0.0027 & 0.0396 & Upregulated \\
\hline hsa-miR-345-3p & 59.74 & 56.13 & 0.0026 & 0.0396 & Downregulated \\
\hline hsa-miR-3622b-5p & 38.67 & 36.86 & 0.0028 & 0.0396 & Downregulated \\
\hline hsa-miR-451a & 25.98 & 32.37 & 0.0029 & 0.0396 & Upregulated \\
\hline hsa-miR-5001-5p & 1180.01 & 1096.07 & 0.0028 & 0.0396 & Downregulated \\
\hline hsa-miR-6511a-5p & 43.38 & 40.66 & 0.0029 & 0.0396 & Downregulated \\
\hline hsa-miR-6724-5p & 886.55 & 827.04 & 0.0027 & 0.0396 & Downregulated \\
\hline hsa-miR-28-5p & 0.65 & 0.97 & 0.0030 & 0.0397 & Upregulated \\
\hline hsa-miR-30b-5p & 17.20 & 19.78 & 0.0030 & 0.0397 & Upregulated \\
\hline $\boldsymbol{R U N X 1}(\mathrm{rs} 8134179)^{1}$ & $\mathrm{TT}(\mathrm{N}=793)$ & $\mathrm{TC} / \mathrm{CC}(\mathrm{N}=317)$ & & & \\
\hline hsa-miR-138-2-3p & 6.29 & 7.08 & 0.0003 & 0.0469 & Upregulated \\
\hline hsa-miR-3177-5p & 4.23 & 4.85 & 0.0004 & 0.0469 & Upregulated \\
\hline hsa-miR-3614-5p & 4.96 & 5.95 & $<.0001$ & 0.0469 & Upregulated \\
\hline hsa-miR-4461 & 3.36 & 4.14 & 0.0003 & 0.0469 & Upregulated \\
\hline hsa-miR-4519 & 7.77 & 8.67 & 0.0003 & 0.0469 & Upregulated \\
\hline hsa-miR-5696 & 6.03 & 7.10 & 0.0004 & 0.0469 & Upregulated \\
\hline hsa-miR-658 & 0.64 & 0.80 & 0.0002 & 0.0469 & Upregulated \\
\hline
\end{tabular}

${ }^{1}$ Data presented is restricted to those with the lowest adjusted $\mathrm{p}$ values. Supplemental Table 1 contains all associations with FDR $<0.09$.

Table 3: Associations between SNPs in TGFß-signaling pathway and miRNA expression in site-specific normal mucosa

\begin{tabular}{|c|c|c|c|c|c|}
\hline \multicolumn{6}{|l|}{ Colon Tissue } \\
\hline miRNA & Mean & Mean & P-values & FDR adjusted $P$ & Direction \\
\hline $\boldsymbol{R} \boldsymbol{U N X} \boldsymbol{2}(\mathrm{rs} 12333172)^{1}$ & $\mathrm{CC} / \mathrm{CT}(\mathrm{N}=626)$ & TT $(\mathrm{N}=36)$ & & & \\
\hline hsa-miR-1226-5p & 54.59 & 79.49 & 0.0015 & 0.0717 & Upregulated \\
\hline hsa-miR-26a-5p & 82.50 & 64.46 & 0.0009 & 0.0717 & Downregulated \\
\hline hsa-miR-30c-1-3p & 11.15 & 15.53 & 0.0012 & 0.0717 & Upregulated \\
\hline hsa-miR-3621 & 37.96 & 53.40 & 0.0014 & 0.0717 & Upregulated \\
\hline hsa-miR-3663-3p & 167.22 & 252.07 & 0.0015 & 0.0717 & Upregulated \\
\hline hsa-miR-3960 & 10454.46 & 20170.24 & 0.0015 & 0.0717 & Upregulated \\
\hline hsa-miR-4316 & 6.93 & 4.87 & 0.0014 & 0.0717 & Downregulated \\
\hline
\end{tabular}

(Continued) 


\begin{tabular}{|c|c|c|c|c|c|}
\hline \multicolumn{6}{|l|}{ Colon Tissue } \\
\hline miRNA & Mean & Mean & P-values & FDR adjusted $P$ & Direction \\
\hline hsa-miR-4498 & 63.88 & 56.98 & 0.0002 & 0.0717 & Downregulated \\
\hline hsa-miR-4516 & 17558.75 & 31136.08 & 0.0015 & 0.0717 & Upregulated \\
\hline hsa-miR-4634 & 326.83 & 612.64 & 0.0013 & 0.0717 & Upregulated \\
\hline hsa-miR-4701-3p & 75.06 & 109.36 & 0.0003 & 0.0717 & Upregulated \\
\hline hsa-miR-4783-3p & 16.70 & 23.43 & 0.0013 & 0.0717 & Upregulated \\
\hline hsa-miR-5739 & 1304.71 & 1856.95 & 0.0012 & 0.0717 & Upregulated \\
\hline hsa-miR-628-3p & 18.91 & 17.63 & 0.0010 & 0.0717 & Downregulated \\
\hline hsa-miR-6723-5p & 135.57 & 197.55 & 0.0009 & 0.0717 & Upregulated \\
\hline hsa-miR-758-5p & 32.86 & 30.14 & 0.0008 & 0.0717 & Downregulated \\
\hline hsa-miR-921 & 21.74 & 18.66 & 0.0012 & 0.0717 & Downregulated \\
\hline $\boldsymbol{E I F 4 E}(\mathrm{rs} 12498533)$ & $\mathrm{AA}(\mathrm{N}=188)$ & $\mathrm{AC} / \mathrm{CC}(\mathrm{N}=473)$ & & & \\
\hline hsa-miR-3180-3p & 16.08 & 18.65 & $<.0001$ & 0.0813 & Upregulated \\
\hline BMPR1B (rs13134042) & GG/GA $(\mathrm{N}=640)$ & AA $(N=22)$ & & & \\
\hline hsa-miR-10a-3p & 3.40 & 6.55 & $<.0001$ & 0.0813 & Upregulated \\
\hline $\boldsymbol{R} \boldsymbol{U N X} \boldsymbol{1}(\mathrm{rs} 8134179)^{1}$ & TT $(\mathrm{N}=463)$ & $\mathrm{TC} / \mathrm{CC}(\mathrm{N}=199)$ & & & \\
\hline hsa-miR-526b-5p & 3.52 & 4.13 & $<.0001$ & 0.0406 & Upregulated \\
\hline hsa-miR-658 & 0.72 & 0.95 & $<.0001$ & 0.0406 & Upregulated \\
\hline hsa-miR-4659b-3p & 3.77 & 4.78 & 0.0002 & 0.0542 & Upregulated \\
\hline \multicolumn{6}{|l|}{ Rectal Tissue } \\
\hline SMAD3 (rs12904944) & GG $(\mathrm{N}=193)$ & GA/AA (N=255) & & & \\
\hline hsa-miR-662 & 38.47 & 42.35 & $<.0001$ & 0.0825 & Upregulated \\
\hline TGFBR1 (rs1571590) & $\mathrm{AA}(\mathrm{N}=301)$ & AG/GG (N=147) & & & \\
\hline hsa-miR-29b-3p & 7.59 & 6.19 & 0.0002 & 0.0825 & Downregulated \\
\hline hsa-miR-29c-3p & 15.03 & 12.35 & 0.0002 & 0.0825 & Downregulated \\
\hline EIF4EBP3 (rs250425) & $\mathrm{CC}(\mathrm{N}=288)$ & CT/TT $(\mathrm{N}=181)$ & & & \\
\hline hsa-miR-4715-5p & 1.79 & 2.68 & $<.0001$ & 0.0825 & Upregulated \\
\hline SMAD7 (rs3736242) & GG/GA (N=423) & AA $(N=25)$ & & & \\
\hline hsa-miR-431-5p & 37.73 & 29.85 & $<.0001$ & 0.0825 & Downregulated \\
\hline PTEN (rs532678) ${ }^{1}$ & $\mathrm{CC}(\mathrm{N}=167)$ & $\mathrm{CT} / \mathrm{TT}(\mathrm{N}=281)$ & & & \\
\hline hsa-miR-10a-3p & 2.55 & 1.86 & 0.0002 & 0.0450 & Downregulated \\
\hline hsa-miR-1203 & 2.03 & 1.55 & 0.0014 & 0.0450 & Downregulated \\
\hline hsa-miR-1261 & 3.26 & 2.61 & 0.0012 & 0.0450 & Downregulated \\
\hline hsa-miR-130b-3p & 3.22 & 2.47 & 0.0002 & 0.0450 & Downregulated \\
\hline hsa-miR-1323 & 3.74 & 2.91 & 0.0008 & 0.0450 & Downregulated \\
\hline hsa-miR-138-2-3p & 5.85 & 4.80 & 0.0018 & 0.0450 & Downregulated \\
\hline hsa-miR-146b-5p & 1.11 & 0.73 & 0.0008 & 0.0450 & Downregulated \\
\hline hsa-miR-3617-5p & 3.28 & 2.63 & 0.0012 & 0.0450 & Downregulated \\
\hline
\end{tabular}




\begin{tabular}{|c|c|c|c|c|c|}
\hline \multicolumn{6}{|l|}{ Colon Tissue } \\
\hline miRNA & Mean & Mean & P-values & FDR adjusted $P$ & Direction \\
\hline hsa-miR-3660 & 3.56 & 2.73 & 0.0003 & 0.0450 & Downregulated \\
\hline hsa-miR-425-3p & 13.76 & 16.55 & 0.0005 & 0.0450 & Upregulated \\
\hline hsa-miR-4433-5p & 18.04 & 20.40 & 0.0010 & 0.0450 & Upregulated \\
\hline hsa-miR-4436a & 4.67 & 3.67 & 0.0005 & 0.0450 & Downregulated \\
\hline hsa-miR-4436b-3p & 6.46 & 5.40 & 0.0011 & 0.0450 & Downregulated \\
\hline hsa-miR-4448 & 8.25 & 7.07 & 0.0017 & 0.0450 & Downregulated \\
\hline hsa-miR-4508 & 51.88 & 57.14 & 0.0012 & 0.0450 & Upregulated \\
\hline hsa-miR-452-5p & 8.70 & 7.25 & 0.0009 & 0.0450 & Downregulated \\
\hline hsa-miR-4657 & 1.60 & 1.16 & 0.0018 & 0.0450 & Downregulated \\
\hline hsa-miR-4665-3p & 104.81 & 119.79 & 0.0012 & 0.0450 & Upregulated \\
\hline hsa-miR-4682 & 0.95 & 0.62 & 0.0018 & 0.0450 & Downregulated \\
\hline hsa-miR-4746-5p & 2.91 & 2.33 & 0.0013 & 0.0450 & Downregulated \\
\hline hsa-miR-4748 & 4.64 & 3.78 & 0.0006 & 0.0450 & Downregulated \\
\hline hsa-miR-4787-3p & 94.67 & 106.09 & 0.0003 & 0.0450 & Upregulated \\
\hline hsa-miR-500a-3p & 2.43 & 1.95 & 0.0007 & 0.0450 & Downregulated \\
\hline hsa-miR-509-5p & 7.57 & 6.81 & 0.0018 & 0.0450 & Downregulated \\
\hline hsa-miR-516b-5p & 5.41 & 4.24 & $<.0001$ & 0.0450 & Downregulated \\
\hline hsa-miR-518a-5p & 4.58 & 3.86 & 0.0013 & 0.0450 & Downregulated \\
\hline hsa-miR-518c-5p & 1.22 & 0.82 & 0.0014 & 0.0450 & Downregulated \\
\hline hsa-miR-519e-5p & 1.93 & 1.32 & 0.0006 & 0.0450 & Downregulated \\
\hline hsa-miR-583 & 3.31 & 2.54 & 0.0018 & 0.0450 & Downregulated \\
\hline hsa-miR-629-3p & 14.57 & 16.06 & 0.0015 & 0.0450 & Upregulated \\
\hline hsa-miR-659-5p & 1.58 & 0.98 & 0.0008 & 0.0450 & Downregulated \\
\hline hsa-miR-766-3p & 26.60 & 29.75 & 0.0017 & 0.0450 & Upregulated \\
\hline hsa-miR-92a-1-5p & 0.50 & 0.33 & 0.0015 & 0.0450 & Downregulated \\
\hline TGFBR1 (rs6478974) & $\mathrm{TT}(\mathrm{N}=150)$ & TA/AA $(\mathrm{N}=298)$ & & & \\
\hline hsa-miR-3917 & 94.31 & 87.13 & $<.0001$ & 0.0825 & Downregulated \\
\hline
\end{tabular}

${ }^{1}$ Data presented is restricted to those with the lowest adjusted $\mathrm{p}$ values. Supplementary Table 2 and 3 contain all associations with FDR $<0.09$.

Table 4: Associations with SNPs in TGFß-signaling pathway and differential expression of colorectal carcinoma and normal mucosa

\begin{tabular}{lccccc}
\hline miRNA & Mean & Mean & P-value & FDR adjusted P & Direction \\
\hline SMAD3 (rs12708492) & CC (N=284) & CT/TT (N=778) & & & \\
hsa-miR-23a-5p & 1.00 & 0.21 & 0.0002 & 0.0820 & Downregulated \\
hsa-miR-30c-2-3p & 0.57 & -0.20 & 0.0003 & 0.0820 & Downregulated \\
hsa-miR-4286 & -198.94 & -9.20 & 0.0005 & 0.0820 & Upregulated \\
& & & & & $($ Continued $)$ \\
\hline
\end{tabular}




\begin{tabular}{|c|c|c|c|c|c|}
\hline miRNA & Mean & Mean & P-value & FDR adjusted $P$ & Direction \\
\hline hsa-miR-4753-5p & 3.88 & 2.04 & 0.0003 & 0.0820 & Downregulated \\
\hline hsa-miR-4755-3p & 0.08 & -1.16 & 0.0005 & 0.0820 & Downregulated \\
\hline hsa-miR-4769-5p & 2.51 & 1.45 & 0.0006 & 0.0820 & Downregulated \\
\hline BMPR2 (rs2228545) & GG $(\mathrm{N}=1001)$ & $\mathrm{GA} / \mathrm{AA}(\mathrm{N}=65)$ & & & \\
\hline hsa-miR-484 & -0.01 & 0.79 & $<.0001$ & 0.0820 & Upregulated \\
\hline NFKB1 (rs230510) & AA $(\mathrm{N}=369)$ & AT/TT $(\mathrm{N}=697)$ & & & \\
\hline hsa-miR-3187-5p & 1.37 & 0.63 & $<.0001$ & 0.0410 & Downregulated \\
\hline hsa-miR-3617-5p & 0.38 & -0.41 & $<.0001$ & 0.0410 & Downregulated \\
\hline hsa-miR-4684-3p & 0.12 & -0.32 & 0.0002 & 0.0410 & Downregulated \\
\hline hsa-miR-6500-3p & 0.44 & -0.33 & 0.0002 & 0.0410 & Downregulated \\
\hline hsa-miR-493-3p & 0.34 & -0.17 & 0.0003 & 0.0492 & Downregulated \\
\hline hsa-miR-3189-5p & 0.43 & -0.34 & 0.0005 & 0.0586 & Downregulated \\
\hline hsa-miR-596 & -0.01 & -0.43 & 0.0005 & 0.0586 & Downregulated \\
\hline hsa-miR-4768-3p & -0.04 & -0.68 & 0.0006 & 0.0615 & Downregulated \\
\hline hsa-miR-4461 & 0.94 & 0.31 & 0.0007 & 0.0638 & Downregulated \\
\hline hsa-miR-302c-5p & 0.63 & 0.27 & 0.0008 & 0.0656 & Downregulated \\
\hline hsa-miR-4296 & 0.62 & 0.31 & 0.0009 & 0.0671 & Downregulated \\
\hline hsa-miR-4675 & 0.57 & -0.22 & 0.0011 & 0.0752 & Downregulated \\
\hline hsa-miR-4251 & 1.52 & 0.93 & 0.0013 & 0.0761 & Downregulated \\
\hline hsa-miR-4458 & -1.46 & -2.15 & 0.0013 & 0.0761 & Downregulated \\
\hline hsa-miR-1276 & 0.34 & -0.07 & 0.0014 & 0.0765 & Downregulated \\
\hline SMAD3 (rs2414937) & $\mathrm{GG} / \mathrm{GC}(\mathrm{N}=1027)$ & $\mathrm{CC}(\mathrm{N}=39)$ & & & \\
\hline hsa-miR-590-5p & -0.14 & 1.65 & $<.0001$ & 0.0820 & Upregulated \\
\hline NFKB1 (rs3821958) & $\mathrm{AA}(\mathrm{N}=352)$ & $\mathrm{AG} / \mathrm{GG}(\mathrm{N}=714)$ & & & \\
\hline hsa-miR-4282 & -1.09 & -0.30 & $<.0001$ & 0.0410 & Upregulated \\
\hline hsa-miR-4684-3p & -0.51 & 0.00 & $<.0001$ & 0.0410 & Upregulated \\
\hline hsa-miR-632 & -0.51 & 0.13 & 0.0003 & 0.0820 & Upregulated \\
\hline RUNX3 (rs6672420) & AA $(\mathrm{N}=296)$ & AT/TT $(\mathrm{N}=770)$ & & & \\
\hline hsa-miR-4261 & 1.38 & 0.51 & $<.0001$ & 0.0820 & Downregulated \\
\hline
\end{tabular}

Table 5: Associations between SNPs in TGFB-signaling pathway and differential expression between colon carcinoma and normal mucosa

\begin{tabular}{lccccc}
\hline miRNA & Mean & Mean & P-values & FDR adjusted P & Direction \\
\hline BMPR1B (rs12508087) & TT (N=378) & TA/AA (N=252) & & & \\
hsa-miR-1182 & 0.29 & 3.37 & 0.0008 & 0.0165 & Upregulated \\
hsa-miR-1224-5p & -127.48 & -16.55 & 0.0008 & 0.0165 & Upregulated \\
hsa-miR-1225-5p & -422.11 & 83.75 & 0.0009 & 0.0165 & Upregulated \\
& & & & & (Continued)
\end{tabular}




\begin{tabular}{|c|c|c|c|c|c|}
\hline miRNA & Mean & Mean & P-values & FDR adjusted $P$ & Direction \\
\hline hsa-miR-1228-3p & -2.66 & 2.94 & 0.0006 & 0.0165 & Upregulated \\
\hline hsa-miR-1233-1-5p & -26.41 & -7.97 & 0.0012 & 0.0165 & Upregulated \\
\hline hsa-miR-1234-5p & -828.58 & -28.93 & 0.0012 & 0.0165 & Upregulated \\
\hline hsa-miR-1268b & -24.76 & 179.09 & 0.0003 & 0.0165 & Upregulated \\
\hline hsa-miR-138-2-3p & -0.75 & -1.86 & 0.0002 & 0.0165 & Downregulated \\
\hline hsa-miR-140-3p & -1.45 & -2.78 & $<.0001$ & 0.0165 & Downregulated \\
\hline hsa-miR-146b-5p & 1.07 & 0.36 & 0.0004 & 0.0165 & Downregulated \\
\hline hsa-miR-150-3p & -31.45 & 11.22 & 0.0007 & 0.0165 & Upregulated \\
\hline hsa-miR-188-5p & -76.36 & 2.88 & 0.0010 & 0.0165 & Upregulated \\
\hline hsa-miR-1915-3p & -668.47 & -162.98 & 0.0003 & 0.0165 & Upregulated \\
\hline hsa-miR-194-3p & -1.66 & -0.88 & 0.0006 & 0.0165 & Upregulated \\
\hline hsa-miR-2861 & -1203.17 & -328.03 & 0.0002 & 0.0165 & Upregulated \\
\hline hsa-miR-3141 & 5.19 & 45.05 & 0.0011 & 0.0165 & Upregulated \\
\hline hsa-miR-345-3p & -1.80 & 7.61 & 0.0008 & 0.0165 & Upregulated \\
\hline hsa-miR-3621 & -3.53 & 0.83 & 0.0011 & 0.0165 & Upregulated \\
\hline hsa-miR-3648 & 38.94 & 92.29 & $<.0001$ & 0.0165 & Upregulated \\
\hline hsa-miR-3656 & -298.14 & 144.78 & 0.0004 & 0.0165 & Upregulated \\
\hline hsa-miR-3665 & -590.36 & -110.88 & 0.0004 & 0.0165 & Upregulated \\
\hline hsa-miR-3940-5p & -127.51 & -9.57 & 0.0008 & 0.0165 & Upregulated \\
\hline hsa-miR-4298 & 12.36 & 42.06 & 0.0009 & 0.0165 & Upregulated \\
\hline hsa-miR-4322 & 3.79 & 16.68 & 0.0003 & 0.0165 & Upregulated \\
\hline hsa-miR-4433-3p & -32.27 & 34.14 & 0.0008 & 0.0165 & Upregulated \\
\hline hsa-miR-4436a & -0.07 & -0.82 & 0.0010 & 0.0165 & Downregulated \\
\hline hsa-miR-4463 & -20.48 & 28.51 & 0.0005 & 0.0165 & Upregulated \\
\hline hsa-miR-4466 & -264.72 & 32.20 & 0.0012 & 0.0165 & Upregulated \\
\hline hsa-miR-4507 & -330.49 & -70.42 & 0.0006 & 0.0165 & Upregulated \\
\hline hsa-miR-4508 & -10.99 & -5.08 & 0.0011 & 0.0165 & Upregulated \\
\hline hsa-miR-4640-5p & 2.32 & 5.29 & 0.0005 & 0.0165 & Upregulated \\
\hline hsa-miR-4687-3p & -435.67 & -17.28 & 0.0006 & 0.0165 & Upregulated \\
\hline hsa-miR-4688 & 1.16 & 5.27 & 0.0004 & 0.0165 & Upregulated \\
\hline hsa-miR-4690-5p & -54.41 & -29.56 & 0.0010 & 0.0165 & Upregulated \\
\hline hsa-miR-4701-3p & -0.09 & 7.90 & 0.0011 & 0.0165 & Upregulated \\
\hline hsa-miR-4707-5p & -7.99 & 7.31 & 0.0006 & 0.0165 & Upregulated \\
\hline hsa-miR-4710 & 0.85 & 5.10 & 0.0011 & 0.0165 & Upregulated \\
\hline hsa-miR-4741 & -91.90 & 96.96 & 0.0009 & 0.0165 & Upregulated \\
\hline hsa-miR-483-5p & 3.36 & 25.50 & 0.0010 & 0.0165 & Upregulated \\
\hline hsa-miR-487b & 0.04 & -0.44 & 0.0011 & 0.0165 & Downregulated \\
\hline hsa-miR-498 & -3.77 & 0.20 & 0.0008 & 0.0165 & $\begin{array}{l}\text { Upregulated } \\
\text { (Continued) }\end{array}$ \\
\hline
\end{tabular}




\begin{tabular}{|c|c|c|c|c|c|}
\hline miRNA & Mean & Mean & P-values & FDR adjusted $P$ & Direction \\
\hline hsa-miR-5196-5p & 6.80 & 16.80 & 0.0006 & 0.0165 & Upregulated \\
\hline hsa-miR-5585-3p & -7.93 & 25.37 & 0.0011 & 0.0165 & Upregulated \\
\hline hsa-miR-572 & -95.60 & -27.10 & 0.0007 & 0.0165 & Upregulated \\
\hline hsa-miR-6068 & -507.73 & -158.29 & 0.0005 & 0.0165 & Upregulated \\
\hline hsa-miR-6069 & -1.29 & 2.00 & 0.0003 & 0.0165 & Upregulated \\
\hline hsa-miR-6076 & -32.27 & -1.35 & 0.0006 & 0.0165 & Upregulated \\
\hline hsa-miR-6083 & -8.29 & -4.69 & 0.0006 & 0.0165 & Upregulated \\
\hline hsa-miR-6088 & -412.01 & 127.38 & 0.0012 & 0.0165 & Upregulated \\
\hline hsa-miR-6089 & -5587.19 & -1383.72 & 0.0010 & 0.0165 & Upregulated \\
\hline hsa-miR-6165 & 17.88 & 87.26 & 0.0008 & 0.0165 & Upregulated \\
\hline hsa-miR-623 & -5.24 & 0.67 & 0.0010 & 0.0165 & Upregulated \\
\hline hsa-miR-638 & -690.46 & -126.32 & 0.0008 & 0.0165 & Upregulated \\
\hline hsa-miR-6511a-5p & 1.31 & 6.67 & 0.0003 & 0.0165 & Upregulated \\
\hline hsa-miR-671-5p & -49.00 & 2.17 & 0.0003 & 0.0165 & Upregulated \\
\hline hsa-miR-6722-3p & -11.44 & 7.03 & $<.0001$ & 0.0165 & Upregulated \\
\hline hsa-miR-6724-5p & -90.62 & 32.59 & 0.0012 & 0.0165 & Upregulated \\
\hline hsa-miR-718 & -17.75 & 1.48 & 0.0004 & 0.0165 & Upregulated \\
\hline hsa-miR-937-5p & -43.41 & 42.52 & 0.0009 & 0.0165 & Upregulated \\
\hline hsa-miR-3937 & -6.32 & 3.66 & 0.0013 & 0.0170 & Upregulated \\
\hline hsa-miR-4734 & -24.78 & -2.09 & 0.0013 & 0.0170 & Upregulated \\
\hline hsa-miR-6073 & -1.63 & -2.34 & 0.0013 & 0.0170 & Downregulated \\
\hline BMPR1B (rs2120834) & $\mathrm{GG} / \mathrm{GC}(\mathrm{N}=545)$ & $\mathrm{CC}(\mathrm{N}=85)$ & & & \\
\hline hsa-miR-4638-3p & -0.02 & 0.66 & $<.0001$ & 0.0813 & Upregulated \\
\hline BMPR2 (rs2228545) $)^{1}$ & $\mathrm{GG}(\mathrm{N}=598)$ & GA/AA $(\mathrm{N}=32)$ & & & \\
\hline hsa-miR-3676-3p & 0.06 & 3.22 & $<.0001$ & 0.0406 & Upregulated \\
\hline hsa-miR-550a-5p & -0.86 & 1.65 & $<.0001$ & 0.0406 & Upregulated \\
\hline EIF4EBP3 (rs250425) ${ }^{1}$ & $\mathrm{CC}(\mathrm{N}=398)$ & $\mathrm{CT} / \mathrm{TT}(\mathrm{N}=235)$ & & & \\
\hline hsa-let-7g-5p & 9.14 & -2.75 & 0.0003 & 0.0406 & Downregulated \\
\hline hsa-miR-103a-3p & 21.18 & 9.39 & 0.0005 & 0.0406 & Downregulated \\
\hline hsa-miR-141-3p & 13.30 & 3.76 & 0.0004 & 0.0406 & Downregulated \\
\hline hsa-miR-15b-5p & 9.35 & 1.64 & $<.0001$ & 0.0406 & Downregulated \\
\hline hsa-miR-16-5p & 16.07 & -0.96 & 0.0004 & 0.0406 & Downregulated \\
\hline hsa-miR-194-5p & -20.67 & -42.84 & 0.0004 & 0.0406 & Downregulated \\
\hline hsa-miR-200c-3p & 36.61 & 6.04 & 0.0003 & 0.0406 & Downregulated \\
\hline hsa-miR-215 & -20.96 & -33.34 & 0.0004 & 0.0406 & Downregulated \\
\hline hsa-miR-30b-5p & 2.26 & -2.26 & 0.0004 & 0.0406 & Downregulated \\
\hline hsa-miR-92a-3p & 62.24 & 45.02 & 0.0005 & 0.0406 & Downregulated \\
\hline hsa-miR-192-5p & -32.40 & -59.23 & 0.0007 & 0.0474 & Downregulated \\
\hline
\end{tabular}

(Continued) 


\begin{tabular}{lccccc}
\hline miRNA & Mean & Mean & P-values & FDR adjusted P & Direction \\
\hline hsa-miR-200a-3p & 5.36 & 2.30 & 0.0007 & 0.0474 & Downregulated \\
hsa-miR-107 & 13.80 & 6.52 & 0.0009 & 0.0488 & Downregulated \\
hsa-miR-20a-5p & 43.71 & 33.81 & 0.0009 & 0.0488 & Downregulated \\
hsa-miR-29a-3p & 58.54 & 37.06 & 0.0008 & 0.0488 & Downregulated \\
hsa-miR-200b-3p & 33.13 & 3.61 & 0.0011 & 0.0497 & Downregulated \\
hsa-miR-27a-3p & 31.16 & 23.35 & 0.0010 & 0.0497 & Downregulated \\
hsa-miR-27b-3p & 8.79 & 3.34 & 0.0011 & 0.0497 & Downregulated \\
\hline
\end{tabular}

${ }^{1}$ Data presented limited to the most significant adjusted $\mathrm{p}$ values; Supplementary Table 4 contains information for all associations with FDR $<0.09$.

Table 6: Associations between SNPs in TGFB-signaling pathway and differential expression between paired rectal carcinoma and normal mucosa

\begin{tabular}{|c|c|c|c|c|c|}
\hline miRNA & Mean & Mean & P-values & FDR adjusted $P$ & Direction \\
\hline SMAD3 $(\mathrm{rs} 12901071)^{1}$ & $\mathrm{AA} / \mathrm{AG}(\mathrm{N}=401)$ & $\mathrm{GG}(\mathrm{N}=35)$ & & & \\
\hline hsa-miR-106b-5p & 8.99 & 10.77 & 0.0062 & 0.0633 & Downregulated \\
\hline hsa-miR-1202 & -87.93 & -307.26 & 0.0050 & 0.0633 & Downregulated \\
\hline hsa-miR-1207-5p & -271.79 & -642.49 & 0.0037 & 0.0633 & Downregulated \\
\hline hsa-miR-1224-5p & -108.00 & -243.68 & 0.0038 & 0.0633 & Downregulated \\
\hline hsa-miR-1226-5p & -3.96 & -12.74 & 0.0049 & 0.0633 & Downregulated \\
\hline hsa-miR-1229-3p & -7.12 & -11.15 & 0.0021 & 0.0633 & Downregulated \\
\hline hsa-miR-1233-1-5p & -26.35 & -52.61 & 0.0061 & 0.0633 & Downregulated \\
\hline hsa-miR-1249 & -1.87 & -8.53 & 0.0023 & 0.0633 & Downregulated \\
\hline hsa-miR-1273c & -0.78 & -5.34 & 0.0020 & 0.0633 & Downregulated \\
\hline hsa-miR-134 & -35.39 & -87.31 & 0.0011 & 0.0633 & Downregulated \\
\hline hsa-miR-149-3p & -2.88 & -7.98 & 0.0046 & 0.0633 & Downregulated \\
\hline hsa-miR-17-5p & 40.01 & 46.53 & 0.0030 & 0.0633 & Upregulated \\
\hline hsa-miR-188-5p & -52.14 & -138.57 & 0.0026 & 0.0633 & Downregulated \\
\hline hsa-miR-197-5p & -238.29 & -716.04 & 0.0036 & 0.0633 & Downregulated \\
\hline hsa-miR-19b-3p & 17.46 & 20.65 & 0.0024 & 0.0633 & Downregulated \\
\hline hsa-miR-20b-5p & 12.23 & 14.48 & 0.0018 & 0.0633 & Upregulated \\
\hline hsa-miR-26b-5p & 0.16 & 2.92 & 0.0037 & 0.0633 & Downregulated \\
\hline hsa-miR-2861 & -1175.18 & -2275.40 & 0.0065 & 0.0633 & Downregulated \\
\hline hsa-miR-29b-3p & 11.43 & 16.18 & 0.0002 & 0.0633 & Upregulated \\
\hline hsa-miR-29c-3p & 1.32 & 5.20 & 0.0017 & 0.0633 & Downregulated \\
\hline hsa-miR-3132 & -0.42 & -4.66 & 0.0022 & 0.0633 & Downregulated \\
\hline hsa-miR-3188 & -35.57 & -68.70 & 0.0059 & 0.0633 & Downregulated \\
\hline hsa-miR-3194-5p & -15.89 & -43.87 & 0.0063 & 0.0633 & Downregulated \\
\hline hsa-miR-3196 & -223.46 & -396.40 & 0.0047 & 0.0633 & Downregulated \\
\hline
\end{tabular}




\begin{tabular}{|c|c|c|c|c|c|}
\hline miRNA & Mean & Mean & P-values & FDR adjusted $P$ & Direction \\
\hline hsa-miR-3197 & -3.13 & -7.12 & 0.0055 & 0.0633 & Downregulated \\
\hline hsa-miR-34a-5p & 8.57 & 11.04 & 0.0012 & 0.0633 & Upregulated \\
\hline hsa-miR-3610 & -20.83 & -45.81 & 0.0049 & 0.0633 & Downregulated \\
\hline hsa-miR-3621 & -3.32 & -8.78 & 0.0038 & 0.0633 & Downregulated \\
\hline hsa-miR-3665 & -533.04 & -1101.36 & 0.0066 & 0.0633 & Downregulated \\
\hline hsa-miR-3682-3p & -1.62 & -12.28 & 0.0063 & 0.0633 & Downregulated \\
\hline hsa-miR-370 & 2.70 & -4.18 & 0.0021 & 0.0633 & Downregulated \\
\hline hsa-miR-371a-5p & -7.65 & -39.77 & 0.0021 & 0.0633 & Downregulated \\
\hline hsa-miR-3917 & -3.25 & -18.21 & 0.0045 & 0.0633 & Downregulated \\
\hline hsa-miR-3925-5p & -4.64 & -10.52 & 0.0064 & 0.0633 & Downregulated \\
\hline hsa-miR-3934-3p & -2.65 & -6.87 & 0.0007 & 0.0633 & Downregulated \\
\hline hsa-miR-3945 & -7.76 & -14.21 & 0.0056 & 0.0633 & Downregulated \\
\hline hsa-miR-4253 & -2.64 & -9.12 & 0.0009 & 0.0633 & Downregulated \\
\hline hsa-miR-4270 & -20.58 & -94.56 & 0.0042 & 0.0633 & Downregulated \\
\hline hsa-miR-4314 & -3.87 & -10.44 & 0.0057 & 0.0633 & Downregulated \\
\hline hsa-miR-4419b & -1.11 & -4.38 & 0.0041 & 0.0633 & Downregulated \\
\hline hsa-miR-4430 & -168.61 & -399.75 & 0.0021 & 0.0633 & Downregulated \\
\hline hsa-miR-4476 & -6.62 & -16.39 & 0.0066 & 0.0633 & Downregulated \\
\hline hsa-miR-4481 & -4.73 & -16.10 & 0.0051 & 0.0633 & Downregulated \\
\hline hsa-miR-4486 & -14.98 & -50.49 & 0.0017 & 0.0633 & Downregulated \\
\hline hsa-miR-4487 & -9.85 & -25.84 & 0.0030 & 0.0633 & Downregulated \\
\hline hsa-miR-4497 & -404.65 & -1071.14 & 0.0006 & 0.0633 & Downregulated \\
\hline hsa-miR-4508 & -11.27 & -19.57 & 0.0058 & 0.0633 & Downregulated \\
\hline hsa-miR-4513 & 0.07 & -3.34 & 0.0057 & 0.0633 & Downregulated \\
\hline hsa-miR-4516 & -3221.62 & -6327.76 & 0.0031 & 0.0633 & Downregulated \\
\hline hsa-miR-4530 & -582.44 & -2083.04 & 0.0007 & 0.0633 & Downregulated \\
\hline hsa-miR-4535 & -5.24 & -13.70 & 0.0043 & 0.0633 & Downregulated \\
\hline hsa-miR-4632-5p & -18.74 & -47.70 & 0.0037 & 0.0633 & Downregulated \\
\hline hsa-miR-4646-5p & -2.94 & -19.35 & 0.0042 & 0.0633 & Downregulated \\
\hline hsa-miR-4655-5p & -0.88 & -6.36 & 0.0013 & 0.0633 & Downregulated \\
\hline hsa-miR-4656 & -2.80 & -21.20 & 0.0044 & 0.0633 & Downregulated \\
\hline hsa-miR-4665-5p & -3.35 & -10.95 & 0.0030 & 0.0633 & Downregulated \\
\hline hsa-miR-4695-5p & -40.01 & -97.81 & 0.0015 & 0.0633 & Downregulated \\
\hline hsa-miR-4721 & -158.17 & -394.68 & 0.0031 & 0.0633 & Downregulated \\
\hline hsa-miR-4734 & -26.33 & -64.44 & 0.0019 & 0.0633 & Downregulated \\
\hline hsa-miR-4739 & -150.29 & -326.46 & 0.0008 & 0.0633 & Downregulated \\
\hline hsa-miR-4740-5p & -1.84 & -7.04 & 0.0022 & 0.0633 & Downregulated \\
\hline hsa-miR-4741 & -61.75 & -305.13 & 0.0028 & 0.0633 & $\begin{array}{l}\text { Downregulated } \\
\qquad(\text { Continued })\end{array}$ \\
\hline
\end{tabular}




\begin{tabular}{|c|c|c|c|c|c|}
\hline miRNA & Mean & Mean & P-values & FDR adjusted $P$ & Direction \\
\hline hsa-miR-4745-5p & -36.68 & -76.35 & 0.0024 & 0.0633 & Downregulated \\
\hline hsa-miR-4758-5p & 2.72 & -16.93 & 0.0061 & 0.0633 & Downregulated \\
\hline hsa-miR-4763-3p & -115.00 & -347.06 & 0.0055 & 0.0633 & Downregulated \\
\hline hsa-miR-4783-3p & -2.21 & -5.03 & 0.0023 & 0.0633 & Downregulated \\
\hline hsa-miR-4788 & -15.89 & -64.61 & 0.0036 & 0.0633 & Downregulated \\
\hline hsa-miR-5001-5p & -220.39 & -447.83 & 0.0063 & 0.0633 & Downregulated \\
\hline hsa-miR-5195-3p & -13.20 & -40.01 & 0.0049 & 0.0633 & Downregulated \\
\hline SMAD3 (rs1498506) & $\mathrm{AA}(\mathrm{N}=120)$ & $\mathrm{AC} / \mathrm{CC}(\mathrm{N}=316)$ & & & \\
\hline hsa-miR-652-3p & 0.05 & 0.63 & $<.0001$ & 0.0825 & Upregulated \\
\hline BMPR2 (rs2228545) & GG $(\mathrm{N}=403)$ & GA/AA (N=33) & & & \\
\hline hsa-miR-324-5p & 1.42 & 3.90 & 0.0002 & 0.0825 & Upregulated \\
\hline hsa-miR-484 & 0.06 & 1.57 & $<.0001$ & 0.0825 & Upregulated \\
\hline SMAD3 (rs2414937) ${ }^{1}$ & GG/GC (N=418) & $\mathrm{CC}(\mathrm{N}=18)$ & & & \\
\hline hsa-miR-1273g-3p & -791.41 & -65.42 & 0.0006 & 0.0550 & Upregulated \\
\hline hsa-miR-17-5p & 41.32 & 22.24 & 0.0003 & 0.0550 & Downregulated \\
\hline hsa-miR-196b-5p & 11.40 & 2.67 & 0.0003 & 0.0550 & Downregulated \\
\hline hsa-miR-20a-5p & 48.80 & 28.38 & 0.0005 & 0.0550 & Downregulated \\
\hline hsa-miR-3651 & 34.71 & 18.55 & 0.0006 & 0.0550 & Downregulated \\
\hline hsa-miR-375 & -46.53 & -24.12 & 0.0005 & 0.0550 & Upregulated \\
\hline hsa-miR-4538 & -42.83 & -10.65 & 0.0004 & 0.0550 & Upregulated \\
\hline hsa-miR-4539 & -23.96 & -5.29 & $<.0001$ & 0.0550 & Upregulated \\
\hline hsa-miR-4697-5p & -19.48 & 13.48 & 0.0003 & 0.0550 & Upregulated \\
\hline $\boldsymbol{R} \boldsymbol{U N X} 2(\mathrm{rs} 2819854)^{1}$ & $\mathrm{CC} / \mathrm{CT}(\mathrm{N}=341)$ & TT $(\mathrm{N}=95)$ & & & \\
\hline hsa-miR-10b-5p & -2.34 & 1.92 & 0.0012 & 0.0715 & Upregulated \\
\hline hsa-miR-1182 & 1.20 & -1.13 & 0.0008 & 0.0715 & Downregulated \\
\hline hsa-miR-1281 & -9.98 & -17.90 & 0.0013 & 0.0715 & Downregulated \\
\hline hsa-miR-129-5p & -1.16 & -2.30 & 0.0008 & 0.0715 & Downregulated \\
\hline hsa-miR-3150b-5p & -3.63 & -6.53 & 0.0013 & 0.0715 & Upregulated \\
\hline hsa-miR-3648 & 45.76 & 9.95 & 0.0009 & 0.0715 & Downregulated \\
\hline hsa-miR-365a-5p & -0.28 & -2.66 & 0.0006 & 0.0715 & Downregulated \\
\hline hsa-miR-3911 & 10.28 & 1.46 & 0.0008 & 0.0715 & Downregulated \\
\hline hsa-miR-4665-3p & -20.84 & -40.12 & 0.0012 & 0.0715 & Downregulated \\
\hline hsa-miR-4767 & -2.92 & -7.01 & 0.0009 & 0.0715 & Downregulated \\
\hline hsa-miR-4769-3p & -2.33 & -4.60 & 0.0009 & 0.0715 & Downregulated \\
\hline hsa-miR-514b-5p & 2.08 & -1.03 & 0.0013 & 0.0715 & Downregulated \\
\hline hsa-miR-5585-3p & 5.26 & -36.48 & $<.0001$ & 0.0715 & Downregulated \\
\hline
\end{tabular}

${ }^{1}$ Data presented for most significant $\mathrm{p}$ values; Supplementary Table 5 contains information for all associations with FDR $<0.09$. 
One set of SNPs and genes associated with expression of miRNAs in normal mucosa consist of eIF4E and TGFB1 and their receptors. These SNPs were previously shown to be associated with colon and rectal cancer risk [4, 6, 7]. eIF4E is essential for ribosomal recruitment and the initiation of translation [33]; eIF4E binds eIF4A and eIF4G to form the eIF4F complex that binds target mRNAs. In this system, eIF4E appears to be key in the regulation of translation initiation, as it physically binds the mRNA and is the least abundant initiation factor [34]; the interface of these genes with miRNAs is supported given the role of miRNAs is posttranscriptional. It has a sole phosphorylation site that interacts with either eIF4G or non-phosphorylated 4EBP1; the PI3-Akt-mTOR pathway phosphorylates 4EBP1 so that it releases eIF4E, allowing binding to eIF4G [34]. Thus eIF4E is important in the convergence of the TGF- $\beta$ and Akt pathways, and its dysregulation is believed to be an important downstream regulator of Akt's action in tumorigenesis [6]. In the canonical TGF- $\beta$ pathway, activated TGF- $\beta$ receptors phosphorylate the c-terminal serine residues of the transcription factors in two different branches of the pathway [35]. In one branch, BMPs, a pleiotropic group of growth factors, are activated and signal through Smads 1/5/8 which act as transcription factors [36]. In the other branch, Smad2 and Smad3 (p-Smad 2/3) form heterotrimeric complexes with Smad 4 , which translocate into the nucleus and regulate target gene expression [37]. PTEN rs532678 was associated with miRNA regulation in normal rectal mucosa. PTEN has been shown previously to influence the TGF $\beta$ pathway through downregulation of miRNAs [30].

The second role of the TGF- $\beta$ signaling pathway, which is associated with greater metastatic potential, includes SNPs in $B M P S, R U N X, S M A D 3$, and $N F \kappa B 1$. In this current study, we show that some of our previously observed SNPs associated with survival, play a role in differential miRNA expression between normal mucosa, colon, and rectal carcinoma tissues. This suggests that the second role of the TGF- $\beta$ signaling pathway, i.e. greater metastatic potential, may be at play. The miRNAs associated with SNPs in SMAD3 and RUNX for differential expression were different from those associated with these SNPs in normal mucosa, further suggesting different mechanisms by which genes and corresponding pathways are being regulated. These genes have previously been shown to play various roles in phenotype aggression, angiogenesis, and metastasis. BMPs are pleiotropic growth factors, whose dysregulation has been shown to deregulate colonic stem cell renewal in mouse models, allowing for de novo crypt formation [36]. In addition to their contribution to tumor initiation, BMP dysregulation has also been shown to play a role in angiogenesis [38, 39]. RUNX2, has been associated with survival and aggressive phenotype in sarcoma [40]. Likewise, in colon cancer, RUNX2 has been significantly associated with Dukes staging, liver metastasis, and ER $\beta$ status [41]. In mice models, SMAD3 loss has been associated with mRNA expression of VEGF, MCP-1, and IL-6 in the choroid [42]. Similarly, in colon cancer these cytokines have been associated with the tumor microenvironment, including angiogenesis. Moreover, in drug resistant colorectal cancer cells 5-flourouracil (5FU) can stimulate Smad3, which is believed to contribute to drug resistance [43]. We have previously shown that interferons, possibly through NF-kB signaling, are associated with colon and rectal cancer risk and survival [44]. It is possible that the diversity of mechanisms associated with these genes and SNPs is through their regulation of miRNAs, which in turn influence expression of hundreds of other genes and pathways.

We have previously reported that $S M A D 3$ was associated with survival in rectal cancer [3]. Of the SMAD3 SNPs associated with differential miRNA expression by genotype, SMAD3 rs12708492 and rs2414937 were previously shown to their influence on survival in rectal and colon tumors cancers [6]. Here we find that rs2414037 is associated with differential miRNA expression between normal mucosa, colon, and rectal carcinoma tissues overall as well as for rectal tumors specifically; suggesting that miRNA dysregulation may be responsible for some of our previously documented observations with regards to the TGF- $\beta$ pathway and survival. RUNX2 also has been found to be associated with colon and rectal cancer. We previously found $R U N X 2$, particularly interactions between these SNPs in RUNX2 and $S M A D 3$, showed strong interaction to increase risk for rectal cancer, as well as prognostic implications [6].

As we have previously shown [4] several SNPs associated with genes in this pathway interact with each other and with lifestyle factors to alter risk of colorectal cancer. Thus, this is a complex pathway. In this study we have only examined the main effects of the SNPs on levels of miRNA expression, although it should be acknowledged that pathways are more complicated given the extensive interaction between SNPs and other genetic and lifestyle factors. Interpretation of results is also complicated by the difficulty in assessing functionality of specific miRNAs. Given that each miRNA can regulated 100 s or even 1000 s of genes, there is a general lack of specificity in determining functionality in any specific setting. It is hoped that over time, as more information on miRNAs become available, determining specific functionality will become more straightforward. Despite these limitations, we believe that the data presented is making strides in understanding how miRNAs work as well as how specific genes in the TGF- $\beta$-signaling pathway operate. The study represents a large collection of colon and rectal data with paired normal and carcinoma tissue. We used the Agilent platform that allowed us to examine almost 1000 miRNAs commonly expressed in colorectal tissue. The data obtained from this platform has been shown to have both high repeatability and validity in terms of comparisons with qPCR expression 
data. Because studies such as the one presented here are relatively few, we encourage others to conduct similar studies to build on and confirm our results.

In summary we have shown that genetic variation in the TGF- $\beta$-signaling pathway influences expression of miRNAs in colorectal tissue. While some genes and their related SNPs influenced miRNA expression in normal tissue, another set influences differential expression between carcinoma and normal mucosa. These results provide support for the functionality of these SNPs that previously have been associated with either colorectal cancer risk or survival, but also provide insight into how expression levels of miRNAs can be altered. The influence on miRNA expression by SNPs in the TGF- $\beta$-signaling pathway and the influence of miRNAs on 100s of targeted genes embody the diversity and importance of the TGF- $\beta$ signaling pathway in the carcinogenic process.

\section{MATERIALS AND METHODS}

\section{Study participants}

Study participants were recruited as part of two population-based case-control studies that included all incident colon and rectal cancer between 30 to 79 years of age who resided in Utah or were members of the Kaiser Permanente Medical Care Program (KPMCP) in Northern California. Participants were white, Hispanic, or black for the colon cancer study and also included participants of Asian race for the rectal portion of the study $[45,46]$. Case diagnosis was verified by tumor registry data as a first primary adenocarcinoma of the colon and were diagnosed between October 1991 and September 1994 and for rectal were diagnosed between May 1997 and May 2001. Detailed study methods have been described [47]. The Institutional Review Boards of the University of Utah and at KPMCP approved the study. In this study we included 1188 participants for whom we have genotype and miRNA data.

\section{miRNA processing}

Formalin-fixed paraffin embedded tissue from the initial biopsy or surgery was used to extract RNA. Carcinoma tissue and adjacent normal mucosa were used to make RNA. Cells were dissected from 1-4 sequential sections on aniline blue stained slides using an H\&E slide for reference. Total RNA was extracted, isolated, and purified using the RecoverAll Total Nucleic Acid isolation kit (Ambion); RNA yields were determined using a NanoDrop spectrophotometer.

\section{miRNA}

The Agilent Human miRNA Microarray V19.0 was used due to the number of miRNAs, its high level of reliability (repeatability coefficient was 0.98 in our data), and the amount of RNA needed to run the platform. The microarray contains probes for 2006 unique human miRNAs as described previously. Data were required to pass stringent QC parameters established by Agilent that included tests for excessive background fluorescence, excessive variation among probe sequence replicates on the array, and measures of the total gene signal on the array to assess low signal. If samples failed to meet quality standards for any of these parameters, the sample was re-labeled, hybridized to arrays, and scanned again. If a sample failed QC assessment a second time, the sample was deemed to be of poor quality and the sample was excluded from analysis. Our previous analysis has shown that the repeatability associated with this microarray was extremely high $(\mathrm{r}=0.98)$ [47], and that comparison of miRNA expression levels obtained from the Agilent microarray to those obtained from qPCR had an agreement of $100 \%$ in terms of directionality of findings and that the fold change calculated for the miRNA expression difference between carcinoma and normal colonic mucosa was almost identical [48]. Of the 2006 unique human miRNAs assessed on the Agilent microarray, 1394 were expressed in colorectal tissue, 1226 in colorectal carcinoma tissue, and 1179 in normal colorectal mucosa. We further restricted the analysis to those miRNAs for which at least $20 \%$ of the population showed expression, leaving 820 miRNAs for colorectal cancer, 813 miRNAs for colon cancer, and 825 miRNAs for rectal cancer for analysis.

To normalize differences in miRNA expression that could be attributed to the array, amount of RNA, location on array, or factors that could erroneously influence miRNA expression levels, total gene signal was normalized by multiplying each sample by a scaling factor [49], which was the median of the $75^{\text {th }}$ percentiles of all the samples divided by the individual $75^{\text {th }}$ percentile of each sample.

\section{TagSNP selection and genotyping}

A customized GoldenGate chemistry (Illumina, San Diego, California) platform was assembled based on genes associated with the CHIEF (Convergence of Hormones, Inflammation, and Energy-related Factors) pathway [7, 26, $32,50]$. TagSNPs for genes in this pathway were selected using the following parameters: an $\mathrm{r}^{2}=0.8$ defined linkage disequilibrium (LD) blocks using a Caucasian LD map, minor allele frequency or minor allele frequency (MAF) $>0.1$, range $=-1500 \mathrm{bps}$ from the initiation codon to +1500 bps from the termination codon, and 1 SNP/LD bin. A genotyping call rate of $99.85 \%$ was attained. Blinded internal replicates represented $4.4 \%$ of the sample set. The duplicate concordance rate was $100.00 \%$. In this analysis we focused on 21 genes, $B M P 1, B M P 2, B M P 4, B M P R 1 A$, BMPR1B, BMPR2, eIF4E, eIF4EBP3, GDF10, MAPK1, MTOR, NFKB1, PTEN, RUNX1, RUNX2, RUNX3, SMAD2, SMAD3, SMAD4, SMAD7, TGFB1, and TGFBR1. 


\section{Statistical methods}

We assessed 80 SNPs in 21 genes that we previously have shown to be associated with colon or rectal cancer risk $[3,6,7,26,32,51]$ in 1188 individuals who also have miRNA expression in normal mucosa and 1141 who have both carcinoma and normal mucosa miRNA expression. Online Supplemental Table 6 show all SNPs assessed. Analyses were run separately for colon and rectal cancer as well as for colorectal cancer combined. Log base 2 transformed miRNA expression level data were used to determine if miRNA expression levels were significantly different by genotype for each SNP. For the SNPs assessed, we used either a dominant or recessive model of inheritance based on previous reports (See Supplementary Table 7). To determine statistical significance, we fit a least-squares linear regression model to the miRNA expression levels and SNPs, adjusting for age at diagnosis, sex, and race/ethnicity. P-values were generated using the bootstrap method by creating a distribution of $10,000 \mathrm{~F}$ statistics derived by resampling the residuals from the null hypothesis of no association between the SNPs and the miRNA (Davison and Hinkley reference), using the 'boot' package in R. Associations were considered important if the false discovery rate (FDR) was less than 0.09 as described by Benjamini and Hochberg [52]; these associations had q-values less than 0.05 [53]. We present those most significant in the text and attach online supplements for those with a FDR of less than 0.09 (Supplementary Tables 1-5). Analyses were run for colorectal normal mucosa (Table 2) as well as for unique association in normal mucosa by sub-site of the tissue, i.e. colon or rectal (Table 3). Similar analysis were run for differential expression (carcinoma - normal) for colorectal cancer overall (Table 4) and for colorectal sub-site (Table 5 for colon and Table 6 for rectal).

\section{ACKNOWLEDGMENTS}

The contents of this manuscript are solely the responsibility of the authors and do not necessarily represent the official view of the National Cancer Institute. We acknowledge Sandra Edwards for data oversight and study management, Dr. Wade Samowitz for slide review, and Michael Hoffman and Erica Wolff for miRNA analysis. We acknowledge Dr. Bette Caan and the staff at the Kaiser Permanente Medical Research Program for sample and data collection.

\section{CONFLICTS OF INTEREST}

The authors declare no conflicts of interest.

\section{FUNDING}

This study was supported by NCI grants CA163683 and CA48998.

\section{Authors' contributions}

MS obtained funding, planned study, oversaw study data collection and analysis, and wrote the manuscript.

AP and AT helped interpret findings and wrote the manuscript.

$\mathrm{JH}$ conducted statistical analysis and managed data.

JS oversaw statistical analysis.

LM assisted with bioinformatics analysis.

RW oversaw laboratory analysis.

All authors approved final manuscript.

\section{REFERENCES}

1. Gordon KJ, Blobe GC. Role of transforming growth factorbeta superfamily signaling pathways in human disease. Biochim Biophys Acta. 2008; 1782:197-228.

2. Hong S, Lee C, Kim SJ. Smad7 sensitizes tumor necrosis factor induced apoptosis through the inhibition of antiapoptotic gene expression by suppressing activation of the nuclear factor-kappaB pathway. Cancer Res. 2007; 67:9577-83.

3. Slattery ML, Lundgreen A, Herrick JS, Wolff RK, Caan BJ. Genetic variation in the transforming growth factor-beta signaling pathway and survival after diagnosis with colon and rectal cancer. Cancer. 2011; 117:4175-83. doi: 10.1002/ cncr.26018.

4. Slattery ML, Lundgreen A, Wolff RK, Herrick JS, Caan BJ. Genetic variation in the transforming growth factorbeta-signaling pathway, lifestyle factors, and risk of colon or rectal cancer. Diseases of the colon and rectum. 2012; 55:532-40. doi: 10.1097/DCR.0b013e31824b5feb.

5. Slattery ML, Lundgreen A, Herrick JS, Kadlubar S, Caan BJ, Potter JD, Wolff RK. Genetic variation in bone morphogenetic protein and colon and rectal cancer. International journal of cancer. 2012; 130:653-64. doi: 10.1002/ijc. 26047.

6. Slattery ML, Lundgreen A, Herrick JS, Caan BJ, Potter JD, Wolff RK. Associations between genetic variation in RUNX1, RUNX2, RUNX3, MAPK1 and eIF4E and riskof colon and rectal cancer: additional support for a TGF-betasignaling pathway. Carcinogenesis. 2011; 32:318-26. doi: 10.1093/carcin/bgq245.

7. Slattery ML, Herrick JS, Lundgreen A, Wolff RK. Genetic variation in the TGF-beta signaling pathway and colon and rectal cancer risk. Cancer Epidemiol Biomarkers Prev. 2011; 20:57-69. doi: 10.1158/1055-9965.EPI-10-0843.

8. Slattery ML, Herrick J, Curtin K, Samowitz W, Wolff RK, Caan BJ, Duggan D, Potter JD, Peters U. Increased risk of colon cancer associated with a genetic polymorphism of SMAD7. Cancer Res. 2010; 70:1479-85. doi: 10.1158/00085472.CAN-08-1792.

9. Yang G, Yang X. Smad4-mediated TGF-beta signaling in tumorigenesis. Int J Biol Sci. 6:1-8. 
10. Piccirillo SG, Vescovi AL. Bone morphogenetic proteins regulate tumorigenicity in human glioblastoma stem cells. Ernst Schering Found Symp Proc. 2006:59-81.

11. Piccirillo SG, Reynolds BA, Zanetti N, Lamorte G, Binda E, Broggi G, Brem H, Olivi A, Dimeco F, Vescovi AL. Bone morphogenetic proteins inhibit the tumorigenic potential of human brain tumour-initiating cells. Nature. 2006; 444:761-5.

12. Anglin I, Passaniti A. Runx protein signaling in human cancers. Cancer Treat Res. 2004; 119:189-215.

13. Zhu BaK, Natasha. (2007). Transforming Growth Factor-B and Cancer: John Wiley \& Sons, Ltd.

14. Blyth K, Cameron ER, Neil JC. The RUNX genes: gain or loss of function in cancer. Nat Rev Cancer. 2005; 5:376-87.

15. Coffman JA. Runx transcription factors and the developmental balance between cell proliferation and differentiation. Cell Biol Int. 2003; 27:315-24.

16. Ito Y, Miyazono K. RUNX transcription factors as key targets of TGF-beta superfamily signaling. Curr Opin Genet Dev. 2003; 13:43-7.

17. Lee KS, Hong SH, Bae SC. Both the Smad and p38 MAPK pathways play a crucial role in Runx2 expression following induction by transforming growth factor-beta and bone morphogenetic protein. Oncogene. 2002; 21:7156-63. doi: 10.1038/sj.onc.1205937.

18. Cameron ER, Blyth K, Hanlon L, Kilbey A, Mackay N, Stewart M, Terry A, Vaillant F, Wotton S, Neil JC. The Runx genes as dominant oncogenes. Blood Cells Mol Dis. 2003; 30:194-200.

19. Ito Y, Osato M, Ito K. RUNX and cancer. Ann Acad Med Singapore. 2003; 32:S6-7.

20. Ge C, Xiao G, Jiang D, Yang Q, Hatch NE, Roca H, Franceschi RT. Identification and functional characterization of ERK/MAPK phosphorylation sites in the Runx2 transcription factor. J Biol Chem. 2009; 284:32533-43.

21. Guo H, Zhang HY, Wang SL, Ye L, Yang GH, Bu H. Smad4 and ERK2 stimulated by transforming growth factor beta1 in rhabdomyosarcoma. Chin Med J (Engl). 2007; 120:515-21.

22. Javelaud DaMA. Smad Signal Transduction. Interplays between the Smad and Map Kinase Signaling Pathways. Protein and Cell Regulation. 2006; 5:317-34.

23. Rajasekhar VK, Holland EC. Postgenomic global analysis of translational control induced by oncogenic signaling. Oncogene. 2004; 23:3248-64.

24. Aggarwal BB, Shishodia S, Takada Y, Jackson-Bernitsas D, Ahn KS, Sethi G, Ichikawa H. TNF blockade: an inflammatory issue. Ernst Schering Res Found Workshop. 2006:161-86.

25. Ahn KS, Sethi G, Aggarwal BB. Simvastatin potentiates TNF-alpha-induced apoptosis through the down-regulation of NF-kappaB-dependent antiapoptotic gene products: role of IkappaBalpha kinase and TGF-beta-activated kinase-1. J Immunol. 2007; 178:2507-16.
26. Slattery ML, Herrick JS, Lundgreen A, Fitzpatrick FA, Curtin K, Wolff RK. Genetic variation in a metabolic signaling pathway and colon and rectal cancer risk: mTOR, PTEN, STK11, RPKAA1, PRKAG2, TSC1, TSC2, PI3K and Akt1. Carcinogenesis. 31:1604-11.

27. Bierie B, Moses HL. Tumour microenvironment: TGFbeta: the molecular Jekyll and Hyde of cancer. Nat Rev Cancer. 2006; 6:506-20. doi: 10.1038/nrc1926.

28. Welch DR, Hurst DR. Unraveling the 'TGF-beta paradox' one metastamir at a time. Breast Cancer Res. 2013; 15:305. doi: 10.1186/bcr3383.

29. Taylor MA, Sossey-Alaoui K, Thompson CL, Danielpour D, Schiemann WP. TGF-beta upregulates miR-181a expression to promote breast cancer metastasis. J Clin Invest. 2013; 123:150-63. doi: 10.1172/JCI64946.

30. Kato M, Putta S, Wang M, Yuan H, Lanting L, Nair I, Gunn A, Nakagawa Y, Shimano H, Todorov I, Rossi JJ, Natarajan R. TGF-beta activates Akt kinase through a microRNAdependent amplifying circuit targeting PTEN. Nat Cell Biol. 2009; 11:881-9. doi: 10.1038/ncb1897.

31. Hata A, Davis BN. Control of microRNA biogenesis by TGFbeta signaling pathway-A novel role of Smads in the nucleus. Cytokine Growth Factor Rev. 2009; 20:517-21. doi: 10.1016/j.cytogfr.2009.10.004.

32. Slattery ML, Wolff RK, Lundgreen A. A pathway approach to evaluating the association between the CHIEF pathway and risk of colorectal cancer. Carcinogenesis. 2015; 36:4959. doi: 10.1093/carcin/bgu213.

33. Zhang J, Babic A. Regulation of the MET oncogene: molecular mechanisms. Carcinogenesis. 2016; 37:345-55. doi: 10.1093/carcin/bgw015.

34. Martinez-Saez E, Peg V, Ortega-Aznar A, MartinezRicarte F, Camacho J, Hernandez-Losa J, Ferreres Pinas JC, Ramon YCS. peIF4E as an independent prognostic factor and a potential therapeutic target in diffuse infiltrating astrocytomas. Cancer Med. 2016. doi: 10.1002/ cam4.817.

35. Demagny $H$, De Robertis EM. Point mutations in the tumor suppressor Smad4/DPC4 enhance its phosphorylation by GSK3 and reversibly inactivate TGFbeta signaling. Mol Cell Oncol. 2016; 3:e1025181. doi: 10.1080/23723556.2015.1025181.

36. Allaire JM, Roy SA, Ouellet C, Lemieux E, Jones C, Paquet M, Boudreau F, Perreault N. Bmp signaling in colonic mesenchyme regulates stromal microenvironment and protects from polyposis initiation. Int J Cancer. 2016; 138:2700-12. doi: 10.1002/ijc.30001.

37. Redshaw N, Camps C, Sharma V, Motallebipour M, Guzman-Ayala M, Oikonomopoulos S, Thymiakou E, Ragoussis J, Episkopou V. TGF-beta/Smad2/3 signaling directly regulates several miRNAs in mouse ES cells and early embryos. PLoS One. 2013; 8:e55186. doi: 10.1371/ journal.pone.0055186. 
38. Su YW, Chung R, Ruan CS, Chim SM, Kuek V, Dwivedi PP, Hassanshahi M, Chen KM, Xie Y, Chen L, Foster BK, Rosen V, Zhou XF, et al. Neurotrophin-3 Induces BMP-2 and VEGF Activities and Promotes the Bony Repair of Injured Growth Plate Cartilage and Bone in Rats. J Bone Miner Res. 2016; 31:1258-74. doi: 10.1002/jbmr.2786.

39. Scarfi S. Use of bone morphogenetic proteins in mesenchymal stem cell stimulation of cartilage and bone repair. World $\mathrm{J}$ Stem Cells. 2016; 8:1-12. doi: 10.4252/wjsc.v8.i1.1.

40. Yang K, Tang XD, Guo W, Xu XL, Ren TT, Ren CM, Wang SD, Bao X, Zhang F, Sun KK. BMPR2-pSMAD1/5 signaling pathway regulates RUNX2 expression and impacts the progression of dedifferentiated chondrosarcoma. Am J Cancer Res. 2016; 6:1302-16.

41. Sase T, Suzuki T, Miura K, Shiiba K, Sato I, Nakamura Y, Takagi K, Onodera Y, Miki Y, Watanabe M, Ishida K, Ohnuma S, Sasaki H, et al. Runt-related transcription factor 2 in human colon carcinoma: a potent prognostic factor associated with estrogen receptor. Int J Cancer. 2012; 131:2284-93. doi: 10.1002/ijc.27525.

42. Iwanishi H, Fujita N, Tomoyose K, Okada Y, Yamanaka O, Flanders KC, Saika S. Inhibition of development of laserinduced choroidal neovascularization with suppression of infiltration of macrophages in Smad3-null mice. Lab Invest. 2016; 96:641-51. doi: 10.1038/labinvest.2016.30.

43. Romano G, Santi L, Bianco MR, Giuffre MR, Pettinato M, Bugarin C, Garanzini C, Savarese L, Leoni S, Cerrito MG, Leone BE, Gaipa G, Grassilli E, et al. The TGF-beta pathway is activated by 5-fluorouracil treatment in drug resistant colorectal carcinoma cells. Oncotarget. 2016; 7:22077-91. doi: 10.18632/oncotarget.7895.

44. Slattery ML, Lundgreen A, Bondurant KL, Wolff RK. Interferon-signaling pathway: associations with colon and rectal cancer risk and subsequent survival. Carcinogenesis. 2011; 32:1660-7. doi: 10.1093/carcin/bgr189.
45. Slattery ML, Potter J, Caan B, Edwards S, Coates A, Ma KN, Berry TD. Energy balance and colon cancer-beyond physical activity. Cancer Res. 1997; 57:75-80.

46. Slattery ML, Caan BJ, Benson J, Murtaugh M. Energy balance and rectal cancer: an evaluation of energy intake, energy expenditure, and body mass index. Nutr Cancer. 2003; 46:166-71.

47. Slattery ML, Herrick JS, Pellatt DF, Stevens JR, Mullany LE, Wolff E, Hoffman MD, Samowitz WS, Wolff RK. MicroRNA profiles in colorectal carcinomas, adenomas and normal colonic mucosa: variations in miRNA expression and disease progression. Carcinogenesis. 2016; 37:245-61. doi: 10.1093/carcin/bgv249.

48. Pellatt DF, Stevens JR, Wolff RK, Mullany LE, Herrick JS, Samowitz W, Slattery ML. Expression Profiles of miRNA Subsets Distinguish Human Colorectal Carcinoma and Normal Colonic Mucosa. Clin Transl Gastroenterol. 2016; 7:e152. doi: 10.1038/ctg.2016.11.

49. Agilent Technologies I. (2013). Agilent GeneSpring User Manual. (Santa Clara, CA: Aglient Technologies Inc).

50. Slattery ML, Fitzpatrick FA. Convergence of hormones, inflammation, and energy-related factors: a novel pathway of cancer etiology. Cancer Prev Res (Phila Pa). 2009; 2:922-30.

51. Curtin K, Wolff RK, Herrick JS, Abo R, Slattery ML. Exploring multilocus associations of inflammation genes and colorectal cancer risk using hapConstructor. BMC Med Genet. 2010; 11:170.

52. Benjamini YH, Y. Controlling the false discovery rate: a practical and powerful approach to multiple testing. Journal of the Royal Statistical Society. 1995; 57:289-300.

53. Storey JD. A direct approach to false discovery rates. Journal of the Royal Statistical Society: Series B. 2002; 64S: 479-98. 